\title{
A study of data from the ElectroMagnetic MultiFrequency method - EMMF
}

\author{
Valdelírio da Silva e Silva and Cícero Régis, \\ Universidade Federal do Pará - UFPA, Brazil, \\ National Institute of Science and Technology of Petroleum Geophysics - INCT/GP.
}

Copyright 2014, SBGf - Sociedade Brasileira de Geofísica.

Este texto foi preparado para a apresentação no VI Simpósio Brasileiro de Geofísica, Porto Alegre, 14 a 16 de outubro de 2014. Seu conteúdo foi revisado pelo Comitê Técnico do VI SimBGf, mas não necessariamente representa a opinião da SBGf ou de seus associados. É proibida a reprodução total ou parcial deste material para propósitos comerciais sem prévia autorização da SBGf.

\section{Abstract}

This work presents an analysis of the data used in the ElectroMagnetic MultiFrequency method, currently under development in Brazil. The data are build from the radial magnetic component of the field of a large loop of current fixed on the surface. Field sections obtained from numerical modeling are studied and compared with a data set from a survey in the Brazilian Southeast. The results show that the data are quite sensitive to the geoelectrical structures of the subsurface.

\section{Introduction}

The Electromagnetic Multifrequency method (EMMF), as described in Machado and Dias (2012), measures the surface radial component of the magnetic field $\left(H_{r}\right)$ of a very large square loop, with side length of a few hundred meters. From these measurements, the method tries to resolve the geoelectric structures under the surface by using a non-linear search process to build sections of two parameters: apparent resistivity, and the so called "polarization parameter", which is a sensitive indicator of inhomongeneities in the geoelectrical environment. The source remains fixed for each survey line. Source-receiver offsets can be as long as $10 \mathrm{~km}$. The frequency range is from $10^{-1} \mathrm{~Hz}$ to $10^{3} \mathrm{~Hz}$. These large offsets and broad frequency range allow the data to be representative of a sizeable section of the subsurface resistivity distribution. The method has been applied in Brazil with encouraging results (Dias et al., 2005, 2006). Figure 1 illustrates the field configuration used in a survey of the method.

The data actually used in the EMMF method are the real and the imaginary parts of the surface radial magnetic component $H_{r}$, normalized by the vertical component of the magnetic field of a vertical magnetic dipole (VMD) with the same dipole moment as the loop, at the same central position, considered in a vacuum. We represent this reference field component as $H_{z}^{0}$. The function used to generate apparent resistivities and polarization parameter sections is then the normalized field $\bar{H}_{r}$ :

$$
\bar{H}_{r}=\frac{H_{r}(r, \omega)}{H_{z}^{0}(r)} \quad H_{z}^{0}=\frac{-m}{4 \pi r^{3}}, .
$$

where $r$ is the source-receiver offset.

This paper presents a study of the data used in the EMMF

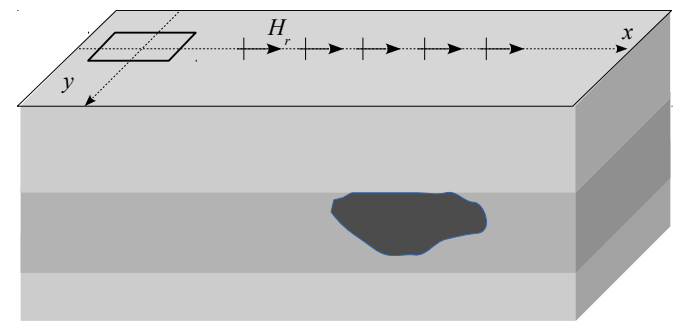

Figure 1: Field configuration of a EMMF survey line.

method, by numerically simulating a survey over a 2D model. Sections of the real and imaginary parts of $\bar{H}_{r}$ are used here to show the main characteristics of this function, and to analise how sensitive it is to the geoelectric structures of the earth. We also present a real data section that exhibit the same general characteristics of our synthetic data.

\section{The field from a dipole source}

Before considering the loop source, let us examine the general solution for the VMD source over a homogeneous half-space. On a typical survey, most, if not all, measurements are taken far enough from the loop source that the EM field is approximated by a dipole field. Therefore, the behavior of the $\bar{H}_{r}$ function for the VMD is relevant to the analysis of the loop response.

The analytical VMD solution for $H_{r}$ (Ward and Hohmann, 1987 ) is written in terms of the modified Bessel functions $\mathrm{I}_{1}, \mathrm{~K}_{1}, \mathrm{I}_{2}$ and $\mathrm{K}_{2}$. The general solution for the normalized $\bar{H}_{r}$ function for this case can be written in terms of the induction number $\theta=r \sqrt{\frac{\omega \mu_{0} \sigma}{2}}$. Making $\Theta=\frac{(1+i)}{2} \theta$, the expression for $\bar{H}_{r}$ is

$$
\bar{H}_{r}=-2 i \theta^{2}\left[I_{1}(\Theta) K_{1}(\Theta)-I_{2}(\Theta) K_{2}(\Theta)\right] .
$$

The real and the imaginary parts of this function are shown in figure 2. These curves are representative of an infinitude of situations for each value of the induction number. Regardless of the configuration, the real part of the function is always negative and has a minimum value at approximately $\theta=3.3$. The imaginary part is negative for small values of the induction number and positive for larger values, with its zero at approximately the same $\theta=3.3$. This implies a maximum of amplitude of $\bar{H}_{r}$ and a phase inversion at the same neighborhood of $\theta$. For a given conductivity, the position of this amplitude peak on the survey line varies with frequency, approaching the source as the frequency rises. 


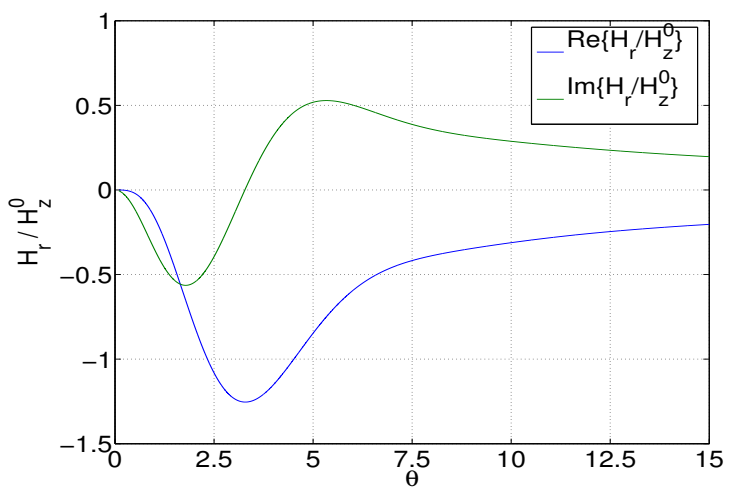

Figure 2: $\bar{H}_{r}$ for a DMV over a homogeneous half-space, as a function of the induction number $\theta$.

As we'll see, the influence of the $2 \mathrm{D}$ anomalous bodies will be felt more strongly around the region of maximum amplitude of $\bar{H}_{r}$.

\section{Synthetic data}

To simulate the EMMF data, we have implemented a Finite Element (FE) code, in a 2.5D formulation based on the one presented in Mitsuhata (2000). The models are composed of a layered medium in which are placed bi-dimensional inhomogeneities infinitely long in the $y$ direction. The method breaks the EM field in two parts: the primary field, which is the field from the source in the background 1D layered model, and the secondary field, which is the difference between the total field in the 2D model and the primary field. The formulation builds a system of differential equations on the electric and magnetic components of the secondary fields on the direction of the strike of the $2 \mathrm{D}$ structures. The source term in these equations are the components of the primary field. This avoids the trouble of representing the singular source on the nodes of the FE mesh. The equations are transformed from the spatial $(x, y, z)$ domain to the spectral $\left(x, k_{y}, z\right)$ domain of the Fourier transform on the strike direction. This transforms the original $3 \mathrm{D}$ problem in a series of $2 \mathrm{D}$ ones on the spectral domain, one for each value of the $k_{y}$ variable. In order to perform the inverse transform back to the spatial domain, we apply a digital filter code that specifies a number of values for the $k_{y}$ coordinates for which the $2 \mathrm{D}$ solution is calculated.

The FE solution gives us the $E_{y}$ and $H_{y}$ components. The $H_{x}$ component on the survey line is derived from these by numerically estimating the directional derivatives needed in Maxwell's equations.

The $2 \mathrm{D}$ model shown in figure 3 is used here to illustrate how the data is sensitive to the variations in the resistivity structure of the subsurface. We simulate a $5 \mathrm{~km}$ survey line, starting at $1.5 \mathrm{~km}$ from the center of the source. The loop radius is $141 \mathrm{~m}$. The results are shown in the sections of figure 4.

The first two images, figures $4 a$ and $4 b$, show the real and the imaginary parts of the data for a homogeneous halfspace with $10 \Omega \cdot \mathrm{m}$, which corresponds to the basement of the model. The contour lines in these figures reproduce the

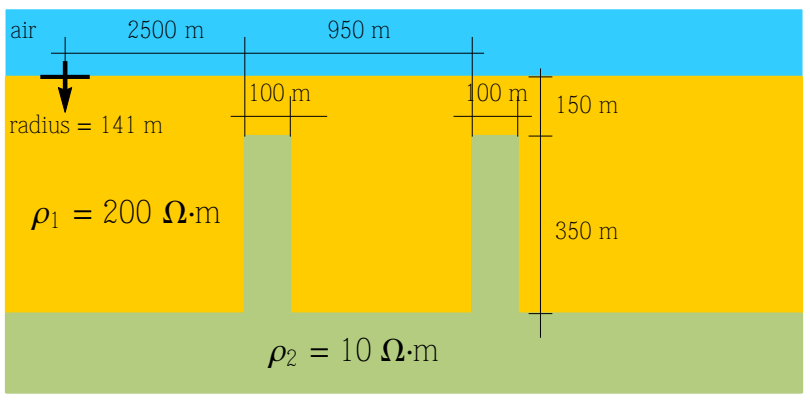

Figure 3: 2D models used to generate the synthetic data.

behavior observed in the curves of figure 2, with the peak of the real part and the zero of the imaginary part located farther from the source in lower frequencies.

When a comparatively more resistive layer $(200 \Omega \cdot \mathrm{m})$ is placed over the homogeneous basement the lines are deformed as observed in figures $4 \mathrm{c}$ and $4 \mathrm{~d}$. The changes in the figures reflect the fact that the data from shorter offsets are more strongly influenced by the top layer, while those at longer offsets suffer more influence from the basement.

The complete model adds two bi-dimensional bodies with the same resistivity as the basement. The presence of the two vertical structures is strongly felt, superimposed on the layered background response, as seen in figures $4 \mathrm{e}$ and $4 \mathrm{f}$. Their lateral positioning is precisely observed. The body closer to the source shows a stronger signal. Otherwise, the responses are similar, their influences extending to the right, at positions farther from the source.

\section{Real data}

The data shown in figure 5 were acquired on the Espírito Santo sedimentary basin, in the Brazilian Southeast. The blank areas are points where data is missing. The source was a square loop with a side length of $600 \mathrm{~m}$ and the survey line is $3 \mathrm{~km}$ long, starting at $1.5 \mathrm{~km}$ from the center of the loop.

Notice the similarity with the results from our layered model. The same general lines are observed, with a slightly more intense response at the beginning of the survey, to the left, which might indicate the presence of lateral variation in the resistivity under this part of the survey line.

\section{Conclusions}

The 2.5D modeling results indicate that the EMMF data is sensitive to both vertical and horizontal variations in the resistivity of the sub-surface under the survey line. Isolated inhomogeneities can be readily identified and a horizontal interface between layers strongly affects the $\bar{H}_{r}$ function.

The similarity of the real data sections to those of the synthetic data suggests that an interpretation of the survey can be made in terms of the same resistivity values used in the model.

The next step in the study of these data is inversion. We have already started this work and we are confident that there will be results to show at this SBGf symposium. 


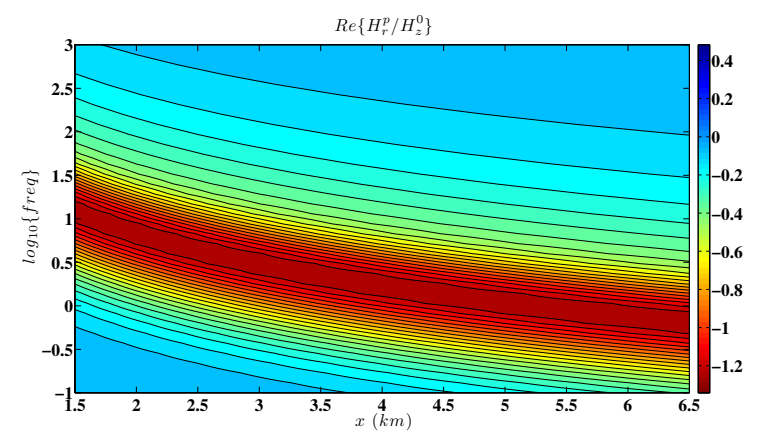

(a) $\operatorname{Re}\left\{\bar{H}_{r}\right\}$ for the $10 \Omega \cdot \mathrm{m}$ homogeneous half-space.

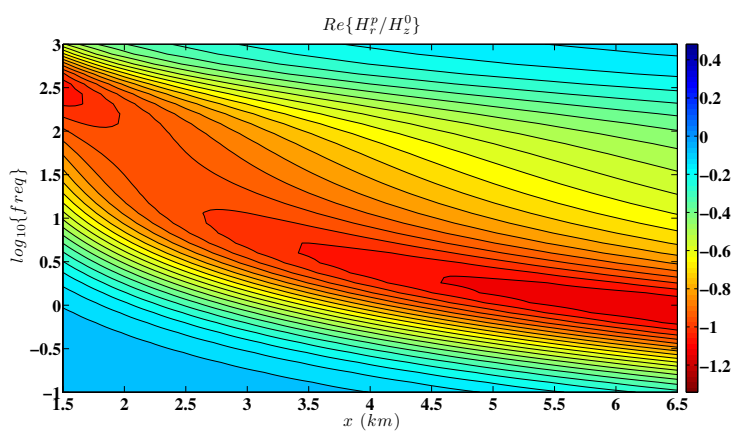

(c) $\operatorname{Re}\left\{\bar{H}_{r}\right\}$ for the two layer model (200 $\Omega \cdot \mathrm{m}$ top layer).

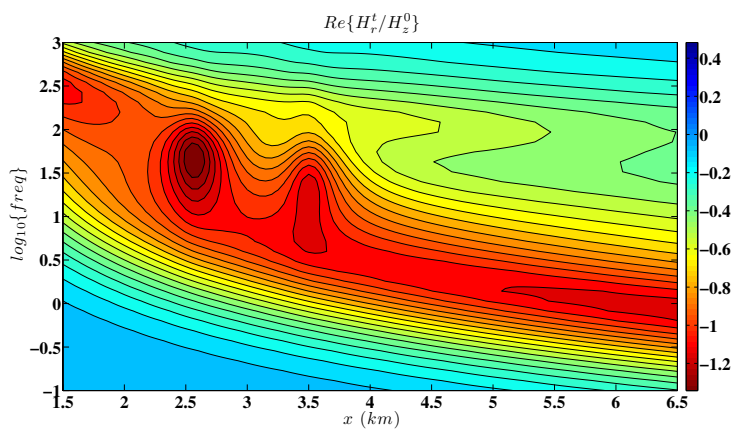

(e) $\operatorname{Re}\left\{\bar{H}_{r}\right\}$ for the complete model.

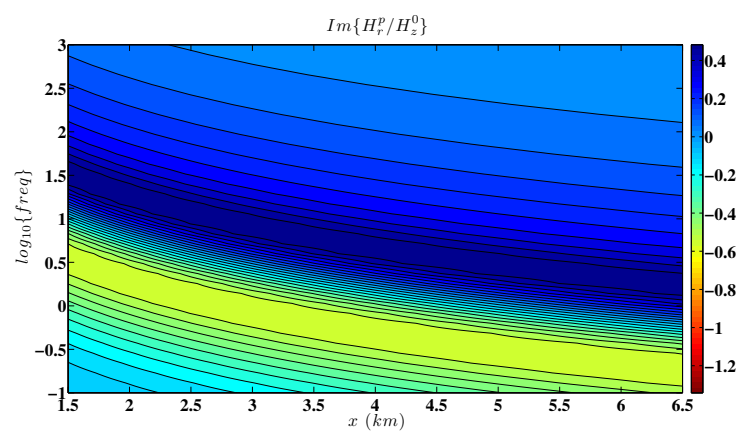

(b) $\operatorname{Im}\left\{\bar{H}_{r}\right\}$ for the $10 \Omega \cdot \mathrm{m}$ homogeneous half-space.

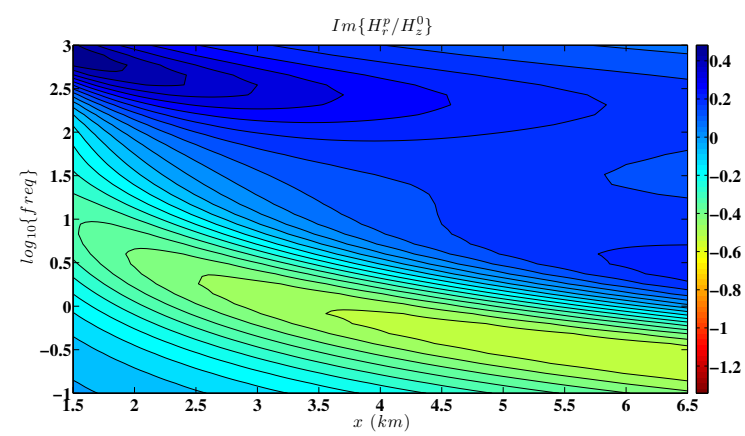

(d) $\operatorname{Im}\left\{\bar{H}_{r}\right\}$ for the two layer model (200 $\Omega \cdot \mathrm{m}$ top layer).

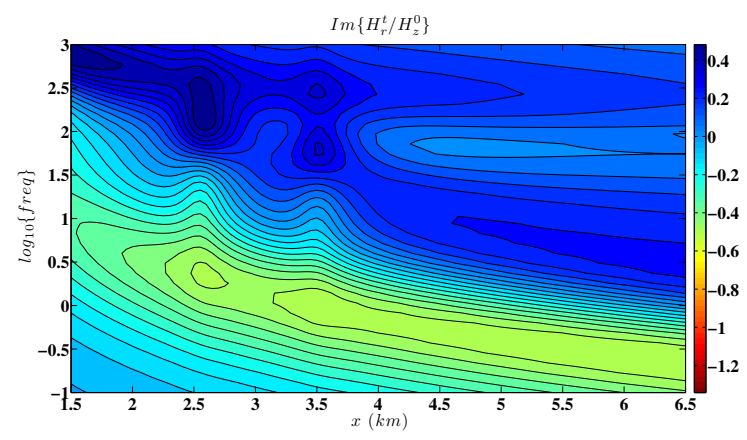

(f) $\operatorname{Im}\left\{\bar{H}_{r}\right\}$ for the complete model.

Figure 4: Synthetic data sections from the model illustrated in figure 3 with a top layer of $200 \Omega \cdot \mathrm{m}$.

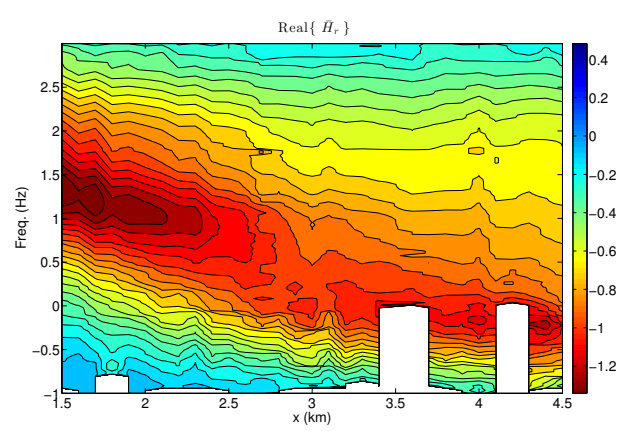

(a) $\operatorname{Re}\left\{\bar{H}_{r}\right\}$.

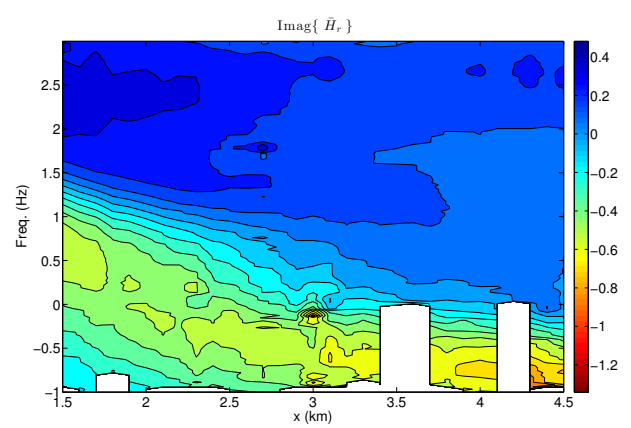

(b) $\operatorname{Im}\left\{\bar{H}_{r}\right\}$.

Figure 5: Real data sections from the Espirito Santo sedimentary basin, in Brazil. 


\section{Aknowledgments}

The authors thank the INCT/GP and the UFPA. Credit is due to the team that acquired the real data, led by professors Hédison Kiuity Sato, Olivar Lima de Lima, both at the CPGG/UFBA, Salvador, and Carlos Alberto Dias at the LENEP/UENF, Macaé, Brazil. This research is partly funded by PETROBRAS, through project number 0050.0056738.10.9-UFBA/FAPEX/PETROBRAS, as part of the Research Network of Applied Geophysics.

\section{References}

Dias, C. A., O. A. L. de Lima Hédison K. Sato, and J. A. C. Moraes, 2006, Contribution to oil exploration and development - a successful inductive multi-frequency em survey on-shore brazil: Presented at the EAGE 68th Conference \& Exhibition, EAGE.

Dias, C. A., H. K. Sato, and O. A. L. de Lima, 2005, Multifrequency em method for hydrocarbon detection and for monitoring fluid invasion during enhanced oil recovery: SEG Technical Program Expanded Abstracts, 24, 602604.

Machado, M. V. B., and C. A. Dias, 2012, Zone of main contribution to the measured signal for a circular current loop source and receiver on the surface of a conductive half-space: Geophysical Prospecting, 60, 1167-1185.

Mitsuhata, Y., 2000, 2-d electromagnetic modeling by finiteelement method with a dipole source and topography: Geophysics, 65, 465-475.

Ward, S. H., and G. W. Hohmann, 1987, 4, in Electromagnetic Theory for Geophysical Applications: SEG, volume 1 of Investigations in Geophysics. 